\title{
Why Are Some Crystals Straight?
}

Chao Li, ${ }^{\dagger}$ Alexander G. Shtukenberg, ${ }^{\dagger}$ Leslie Vogt-Maranto,${ }^{\dagger}$ Efi Efrati,${ }^{\ddagger}$ Paolo Raiteri, ${ }^{\S}$ Julian D. Gale, ${ }^{\S *}$ Andrew L. Rohl ${ }^{I^{* *}} \&$ Bart Kahr ${ }^{\dagger *}$

${ }^{\dagger}$ Department of Chemistry New York University, New York City, New York 10003, United States.

${ }^{\ddagger}$ Department of Physics of Complex Systems, Weizmann Institute of Science, Rehovot 76100, Israel.

${ }^{\S}$ Curtin Institute for Computation and School of Molecular and Life Sciences, Curtin University, P. O. Box U1987, Perth, Western Australia 6845, Australia.

${ }^{\mathrm{II}}$ Curtin Institute for Computation and School of Electrical Engineering, Computing and Mathematical Sciences, Curtin University, P. O. Box U1987, Perth, Western Australia 6845, Australia.

Abstract: More than one quarter of molecular crystals that can be melted, can be made to grow in the form of twisted lamellae or fibers. The mechanisms leading to such unusual crystal morphologies lacking long-range translational symmetry on the mesoscale are poorly understood. Benzil $\left(\mathrm{C}_{6} \mathrm{H}_{5} \mathrm{C}(\mathrm{O})-\mathrm{C}(\mathrm{O}) \mathrm{C}_{6} \mathrm{H}_{5}\right)$ is one such crystal. Here, we calculate the morphology of rod-shaped benzil nanocrystals, and other related structures. The ground states of these ensembles were twisted by 0.05-0.75 degrees/A for rods with cross sections of $50-10 \mathrm{~nm}^{2}$, respectively, the degree of twisting decreased inversely proportional to the crystal cross sectional area. In the aggregate, our computational studies, combined with earlier observations by light microscopy, suggest that in some cases very small crystals acquire 3D translational periodicity only after reaching a certain size. Twisting is accompanied by conformational changes of molecules on the $\{10 \overline{1} 0\}$ surfaces of the six-sided rods, although whether such changes are causes of the twisting, consequences of surface stress where symmetry is broken, or consequences of intrinsic dissymmetry when two or more geometric tendencies are in conflict, is not easily answered from our data. Nevertheless, it has become clear that in some cases, the development of a crystal with a lattice having long range translational symmetry is not foretold in the thermodynamics of aggregates of molecules. Rather, a lattice is sometimes a device for allowing a growing crystal to take advantage of the thermodynamic driving force of growth, a best compromise for a large number of molecules, which on a smaller scale would be 
dissymmetric (have a point symmetry only). The relationship between these calculations and ubiquity of crystal twisting on the mesoscale is discussed.

\section{INTRODUCTION}

Well-formed crystals, whether periodic or even quasi-periodic, are polyhedra with flat faces and sharp edges. While the official definition of crystal since 1992 is based on the sharpness of its diffraction pattern, ${ }^{1}$ a still serviceable part of any colloquial definition of crystal, even if not officially, is this: Crystals are straight systems of points, approximated as lattices, related to one another by translation.

Researchers have recently taken to bending or twisting as-grown molecular crystals by applying forces with pincers ${ }^{2,3}$ or by imposing photochemical ${ }^{4,5,6,7,8}$ and/or thermochemical ${ }^{9}$ transformations. ${ }^{10,11}$ The considerable elasticity and plasticity of molecular crystals, ${ }^{12}$ previously presumed to be brittle, was surprising a decade ago, but the stream of examples since that time has changed the outlook of the scientific community with respect to the mechanical properties of crystals made from molecules. However, long before this recent trend in crystal chemistry and physics, it had been shown that a large fraction of molecular crystals can develop helicoidal morphologies during growth. ${ }^{13,14,15,16}$ In such cases, there are no external forces applied, from forceps, or electrodynamic fields, or from the strain induced by heterogeneous chemical reactions. Of foremost concern to contemporary research is the cause of twisting, the forces involved, as well as the troubling fact that helicoids have curvature; they are not polyhedra, and they are decidedly not straight. ${ }^{17}$ If so many crystals are helicoids, what then is a "crystal"?

Isolated micron- and macro-sized crystals can be twisted, ${ }^{18,19}$ but the vast majority of twisted molecular crystals are found in so-called banded spherulites ${ }^{20,21,22}$ formed by tightly-packed fibrous or lamellar crystals with high aspect ratios. In fact, Bernauer claimed that at least a quarter of molecular crystals can be grown from the melt as aggregates of needle-like crystals twisting more or less in phase. ${ }^{16}$ In our experience, with substances that can be crystallized from the melt, this is an underestimate. Subsequently, it was suggested that as many as one half of synthetic polymers can crystallize as spherulites with twisted fibers. ${ }^{17}$ 
Twists in the crystals of spherulites strongly depend on the growth temperature, primarily because the driving force for crystallization and properties of materials are highly temperature dependent. Typically, higher supercooling and smaller cross-sectional areas drive twisting. ${ }^{17,23,24}$ The twisting of single crystals has been observed with a light microscope in rare cases. For instance, as a needle of hippuric acid grows, twist is mostly manifest at the tip. As the needle thickens, the crystal gradually unwinds. ${ }^{25,26,27}$

The aforementioned evidence prompts a general question: Is translational symmetry a necessary feature of very small crystals, and when can a crystal be adequately described by space group symmetry with long range translations consistent with a lattice? When, then, do molecular crystals generally earn their translational symmetry?

Here, we report that molecular nanorods of benzil, among other small in silico models of crystals of simple molecules, spontaneously twist during force field minimization and molecular dynamics (MD) simulation. The relationship between the twist rate and crystal size is reported and compared with geometrical scaling laws. ${ }^{28}$

\section{RESULTS}

Twisted benzil nanocrystals. The crystal structure of benzil (Figure 1a), space group $P 3_{1(2)} 21$, was first determined in was first considered in the $1920 \mathrm{~s}^{29,30}$ and later refined. ${ }^{31,32,33}$ The three molecules in the unit cell pack around the $3_{1(2)}$ axis with herringbone interactions between the aromatic rings of neighboring molecules (Figure 1b). Large benzil single crystals with a hexagonal bipyramidal or biprism morphology can be obtained by slow evaporation from acetone solutions ${ }^{34}$ or by seeding the melt ${ }^{35}$ (Figure 1f). Large benzil microcrystals were obtained by precipitation from ethanol and water (1:10 v:v) solutions (Figure 1e). Fine benzil fibers, twisted with a mesoscale pitch (crystal length needed to achieve $2 \pi$ rotation, $P \approx 0.6 \mathrm{~mm}$ ) are organized as spherulites. ${ }^{23}$ The periodic, dark extinction bands between crossed polarizers (two bands for $2 \pi$ rotation) are the tell-tale signatures of fibril twisting (Figure 1d). Such banded polycrystalline ensembles can be grown at room temperature in the form of spherulites in the presence of colophony, gum mastic, castor oil, or even without any additives if crystallized below room temperature. Natural oils or resins have the effect of inhibiting nucleation and increasing the effective crystallographic driving force that can otherwise only be achieved by increasing the undercooling of the melt. Under the working hypothesis that benzil microcrystals 
inherit their morphologies from nanocrystals, Figure 1e, albeit by some mechanism not yet clarified, we built a six-sided benzil nanocrystal from the crystal coordinates. ${ }^{32}$ In the course of iterative geometry optimizations and MD cycles (see details in the Methods section below), a crystallographically straight benzil nanocrystal in space group $P 3_{1} 21$ adopted a pronounced lefthanded twist (Figure 1c). A similar twist was also observed under energy minimization alone, illustrating that twisting in this case is a non-activated, barrier-free process. However, the iterative process of geometry optimization followed by molecular dynamics at $10 \mathrm{~K}$ until the optimization criteria were met was used for all subsequent calculations. This was found to speed up the convergence to the final structure. A right-handed twisted crystal was observed for the enantiomorph $P_{2} 21$ (Figure S1), as required by symmetry. The twist rate was quantified on the basis of the positions of the centers of mass of the molecules (Figure S2).
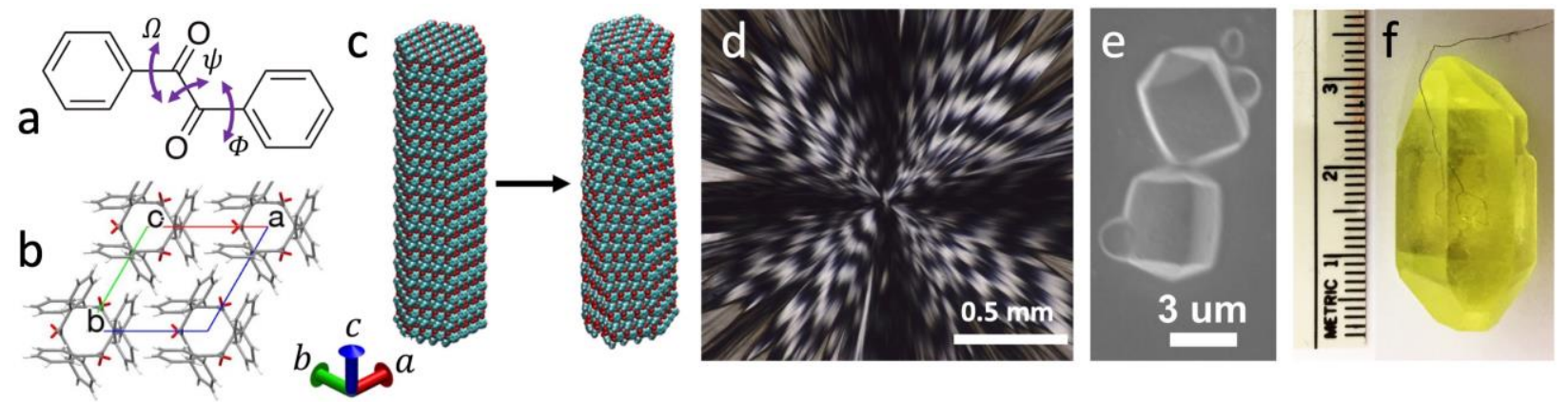

Figure 1. Benzil and its crystals at different length scales. (a) Molecular structure, indicating the three torsional degrees of freedom. (b) Crystal structure $\left(P 3_{1} 21\right)$ viewed along $c$. (c) Trigonal benzil nanocrystalline rod before (left) and after our geometry optimization procedure (right). (d) Optical micrograph of benzil banded spherulites grown from the melt containing $\mathrm{ca} .20 \mathrm{wt}$ \% colophony after quenching in dry ice (growth temperature 0 to $-20{ }^{\circ} \mathrm{C}$ ) and allowing the material to warm to room temperature. Individual crystalline fibrils are no thicker than several microns. (e) SEM image of six-sided benzil microcrystals grown from the mixture of ethanol and water (1:10). (f) Large, benzil single crystal. Metric scale in $\mathrm{cm}$. 
Figure 1d shows banded spherulites of benzil with periodic changes in refractivity. This means that at the mesoscale, the growth direction cannot be $<0001\rangle$. The growth direction is the twisting axis. If the twisting axis were <0001>, directions with the same refractivity, perpendicular to the optic axis, would be modulated. There would be no periodic changes in light intensity between crossed polarizers. For this reason, we also simulated a benzil rod $17 \times 4 \times 3$ unit cells, elongated along $\langle 10 \overline{1} 0\rangle$. The twist of $0.38 \%$ is shown in Figure 2. We now continue with a fuller analysis of $<0001>$ rods as in Figure 1c.

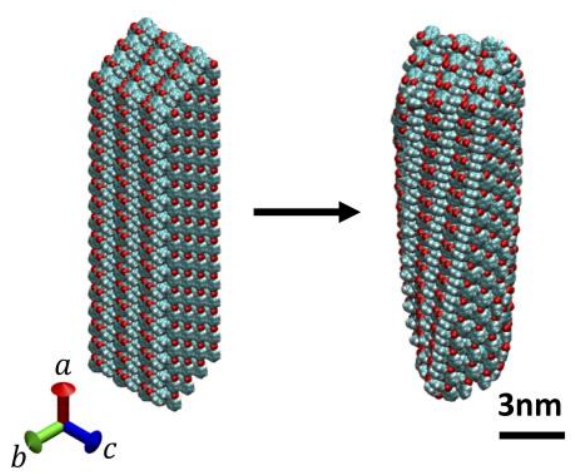

Figure 2. Benzil rod of $17 \times 4 \times 3$ unit cells elongated along $\langle 10 \overline{1} 0\rangle$ based on the crystal structure (left) and twisted after optimization (right).

Effect of crystal size. The relationship between size and twist was evaluated by simulations on benzil $c$-elongated nanocrystals with cross sections of $2,3,4,5$, and 6 unit cells along a $\{10 \overline{1} 0\}$ side of a six-sided prism. The length of the nanocrystals was constant at 15 unit cells. The twist rate, $\theta[\% \AA \AA$ ] did not show strong dependence on the crystal length (Figure S3). Equilibrated structures, twisting angles $(\theta)$, and widths $(w)$ are shown in Figure 3a. Crystals untwist as they get thicker in accordance with the relationship (Figure 3b),

$$
P=2 \pi l \theta=k w^{2}
$$

where $P$ is the pitch (crystal length needed to achieve $2 \pi$ rotation) and $k=1.24 \AA^{-1}$ is a constant.

The potential energy change accompanying twisting was computed by imposing predefined twists on two benzil nanocrystals (measuring 10 unit cells in length, with 4 unit cells on a side) in the General Lattice Utility Program (GULP), ${ }^{36,37}$ and the induced strain was locally optimized using a conjugate gradient minimizer (Figure 3c). Equivalent structures were obtained by minimizing in the absence of dynamics in most cases. The negative twist was assigned to left- 
handed nanocrystals, while positive values corresponded to a right-handed ones. The lowest energy corresponded to the twist rate $c a .4 \%$ unit cell for the $4 \times 4 \times 10$ benzil nanocrystals. Thus, the left-handed twisted structure is more stable than the straight one and there is no energy barrier associated with the transition from a straight crystal to the twisted minimum. In agreement with Figure 2a, these data show that the thicker the crystal, the less it is twisted. The pitch grows, and the crystal unwinds, in proportion to the cross-sectional area. 

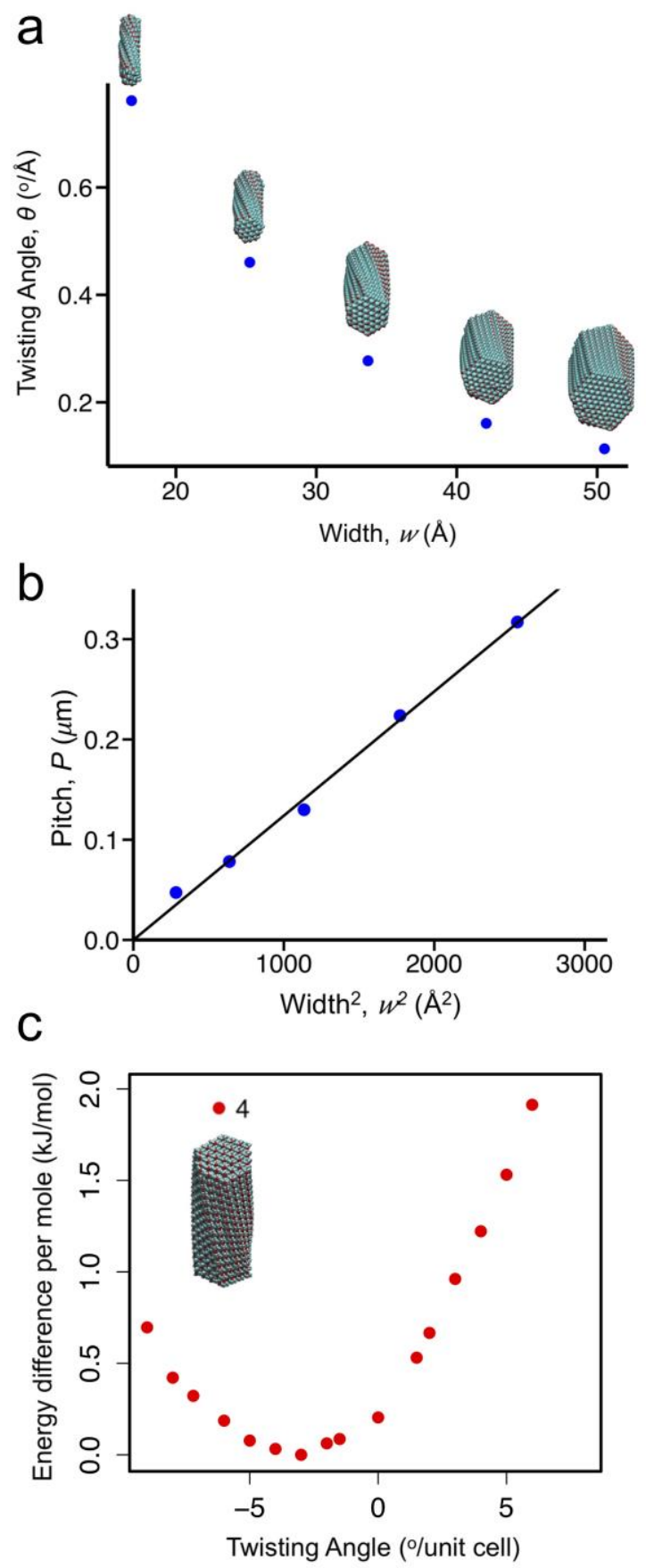

Figure 3. Relationship between twist and size of the crystal. (a) Twisting angle, $\theta$, for spontaneously twisted benzil nanocrystals 15 unit cells long. The sides of the six-sided rod, $w$, ranged from 2-6 unit cells in increments of one cell. (b) Pitch of the twisted benzil nanocrystal $v s$. square of side width, $w^{2}$. (c) Potential energy per molecule calculated for twisted benzil nanocrystals with 4 unit cells on sides. The energy difference was calculated with respect to the lowest energy point for each nanorod. Negative twist correspond to the left-handed twist.

Surface reconstruction in benzil nanocrystals. Benzil has a skew, $C_{2}$-symmetric ${ }^{38,39,40}$ ground state in the gas phase with an $\mathrm{O}=\mathrm{C}-\mathrm{C}=\mathrm{O}$ torsion angle $(\Psi)$ of $117^{\circ 41}$ by electron diffraction and 
$127^{\circ}$ by density functional theory according to the literature, ${ }^{42,43}$ though we have computed a more accurate value of $116.3^{\circ}$. In a periodic crystal, each benzil molecule has an approximate $C_{2}$-symmetric skew configuration (the molecules sit on general positions) with an $\mathrm{O}=\mathrm{C}-\mathrm{C}=\mathrm{O}$ torsion angle $(\Psi)$ of $107^{\circ}$. The value of $\Psi$ following periodic GAFF geometry optimization was $114^{\circ}$. To analyze the distribution of molecular benzil configurations in twisted nanocrystals, we determined the torsion angles $\Omega$ and $\Phi$ of the phenyl rings around the carbonyl group, as well as $\Psi$ (see Figure 1a for illustration of these angles). A benzil nanocrystal measuring $4 \times 4 \times 15$ unit cells was studied in greatest detail. Since the configuration of the molecule may also be influenced by surface reconstruction, the molecules were divided into three groups based on their positions in the crystal (Figure 4a). Except for several molecules on the $\{0001\}$ surfaces, the torsion angles $\Omega$ and $\Phi$ do not significantly change over the crystal (Figure $4 \mathrm{~b}, \mathrm{c}$ ), meaning that the phenyl rings remain nearly coplanar with the $\mathrm{C}=\mathrm{O}$ bonds. The distribution of torsion angles, $\Psi$, however, is much wider. There is a noticeable population of molecules in the outer layer having two $\mathrm{C}=\mathrm{O}$ bonds $\mathrm{ca} .30^{\circ}$ closer to each other than the molecules in the original crystal structure (Figure 4d). This suggests that the possible reason for benzil twisting - or a consequence of twisting - is reconstruction of the $\{10 \overline{1} 0\}$ surfaces. Figure $4 \mathrm{f}$ shows the benzil rod with the surface molecules having the small $\mathrm{O}=\mathrm{C}-\mathrm{C}=\mathrm{O}$ angles in the bimodal distribution of Figure $4 \mathrm{~d}$ marked in brown. It is obvious that the large conformational changes are not manifest in all molecules, and they tend to occur in rows. In other words, the conformational changes appear to be coupled or cooperative.

To test the connection between surface reconstruction and bulk nanocrystal twisting, our geometry optimization procedure was performed using a force field in which, the torsion parameter in the force field for the $\mathrm{O}=\mathrm{C}-\mathrm{C}=\mathrm{O}$ angle, $K$, was stiffened beyond a physically realistic expectation (see Methods section). With the optimized torsion constant $K=0.013 \mathrm{eV}$ given by the GAFF force field, ${ }^{44,45} \Psi$ was broadly distributed between $75^{\circ}$ and $125^{\circ}$ (Figure 4e). As the force constant increases to a value characteristic of a more rigid aromatic system, $K=$ $0.157 \mathrm{eV}$, the distribution became narrower and symmetric around $\Psi=107^{\circ}$, the value corresponding to the starting bulk crystal structure of benzil. This increase in $K$ was accompanied by a decrease in $\theta$ from 3.4 to $1.1 \%$ A (Figure S4). 


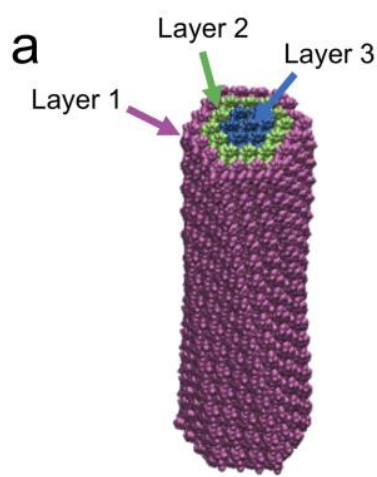

b

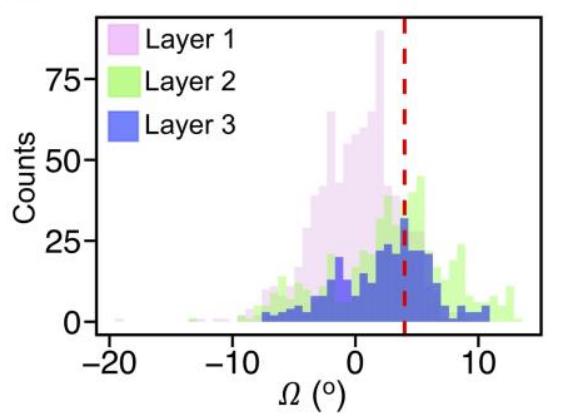

C

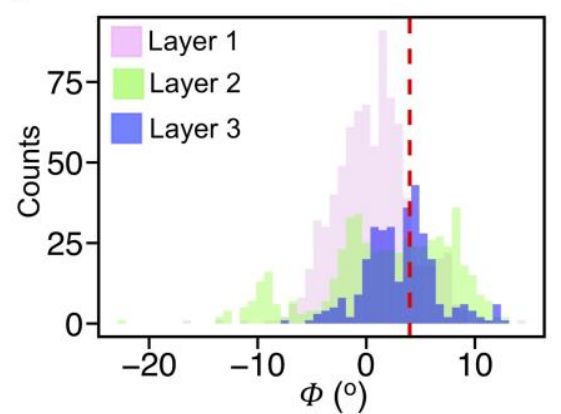

f
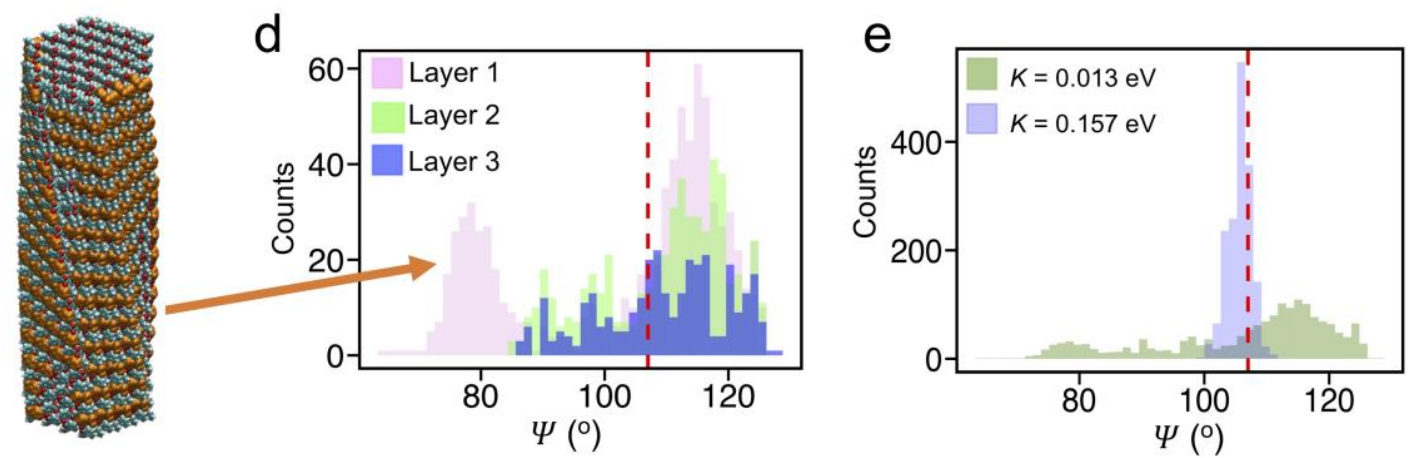

Figure 4. Molecular geometries of twisted benzil crystals. (a) Representation of three layers (layers 1, 2, and 3) from the outside to the center in a benzil nanocrystal measuring 4 unit cells on a side and 15 unit cells in length

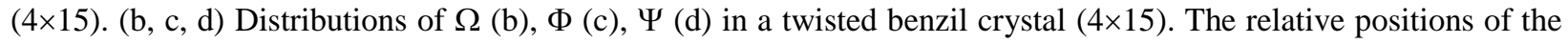
molecules are distinguished by colors. (e) Distributions of $\Psi$ for all molecules in crystals under different force constants $K$. The red dashed lines represent values of corresponding angles in the ideal periodic crystal structure. (f) Twisted benzil nanocrystal with the molecules having smaller $\mathrm{O}=\mathrm{C}-\mathrm{C}=\mathrm{O}$ angles in brown.

Benzil is not unique, but not wholly representative either. Needless to say, benzil nanocrystals are not the only ones that noticeably forgo their space group symmetry. Moreover, some nanocrystals as analyzed above remain straight. 4-Phenoxyaniline $\left(\mathrm{C}_{12} \mathrm{H}_{11} \mathrm{NO}\right.$, space group $P 2{ }_{1}{ }^{46}$ Figure 5a,b,c) is twisted around the $b$ axis, whereas 4,4 '-dimethylbenzophenone $\left(\mathrm{C}_{15} \mathrm{H}_{14} \mathrm{O}\right.$, space group $P 3_{2} 21,{ }^{47}$ Figure 5 d,e,f) barely departs from its crystallographic structure at the nanoscale.

Figure 5b shows a nanocrystal of 4-phenoxyaniline measuring 3, 4, and 20 unit cells along $a, c$, and $b$, respectively, that adopted a right-handed twist after minimization. The twisted geometry was lower in internal energy (Figure 5c). Moreover, the conformations of molecules on the surfaces were of altered conformation, as was the case for benzil (Figure S5A). There was a large proportion of molecules on the surface having the rotation angles between two phenyl rings $c a$. $5^{\circ}$ smaller than the average of the interior molecules. 
a<smiles>Nc1ccc(Oc2ccccc2)cc1</smiles>

b

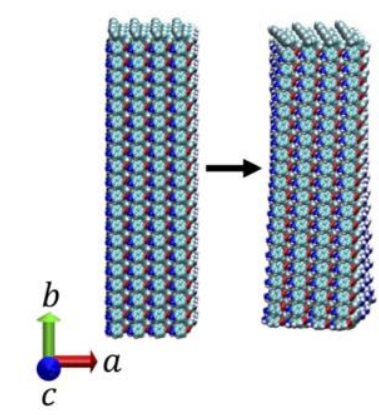

C

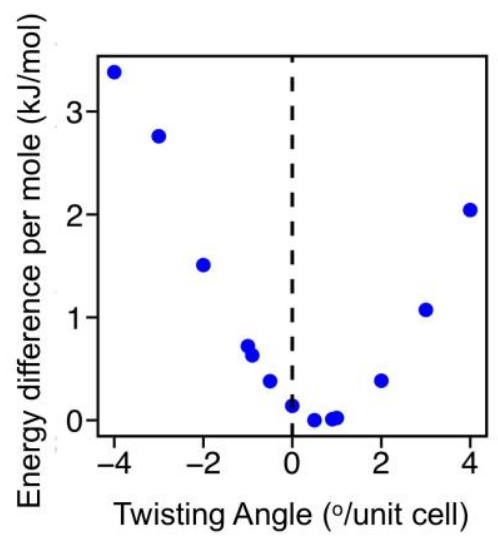

d<smiles>Cc1ccc(C(=O)c2ccc(C)cc2)cc1</smiles>

e

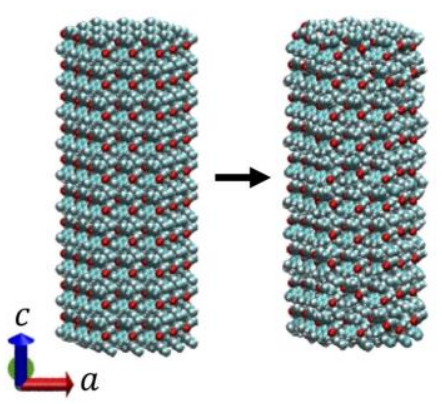

f

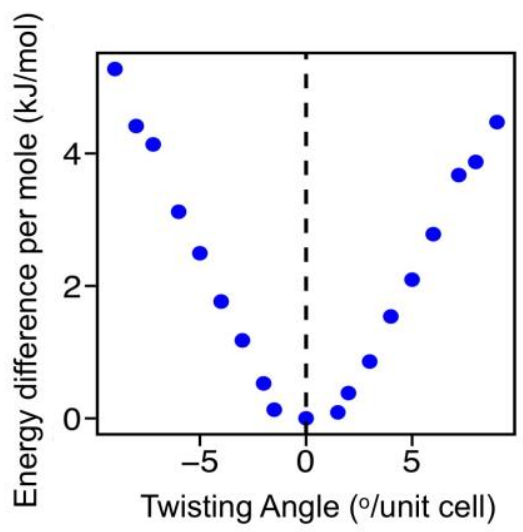

Figure 5. Spontaneously twisted nanocrystals of 4-phenoxyaniline (a-c) and non-twisted crystal of 4,4'dimethylbenzophenone (d-f). (a) and (d) Molecular structures. (b) Nanocrystals of 4-phenoxyaniline show twist after relief of local strain, while (e) nanocrystals of 4,4'-dimethylbenzophenone remained straight. (c) and (f) Energy difference with respect to the lowest energy points per molecule of nanocrystals as functions of twisting angle.

Figure $5 \mathrm{f}$ shows the potential energy of 4,4'-dimethylbenzophenone (Figure $5 \mathrm{~d}$ ) as a function of twist centered at $0^{\circ}$, the crystallographic configuration (Figure 5e). No significant conformational change for molecules on the surface was observed, further demonstrating that twisting and surface distortion are coupled with each other (Figure S5B).

\section{DISCUSSION}

Why are some nanocrystals twisted, lacking long-range translationally symmetry? Uncompensated bonds on the crystal surface are responsible for the surface stresses that may 
drive bulk distortions and morphological changes. ${ }^{48,49}$ Alternatively, a crystal may be viewed as a geometrically frustrated assembly, ${ }^{28,50,51,52,53}$ in which intrinsic distortions of the bulk could affect the crystal morphology and drive conformational changes on the surface. Are the geometric changes we see in molecules on the $\{10 \overline{1} 0\}$ surfaces of benzil causes or consequences of the twisting or both phenomena are simply coincidental? Changes in the surface structure and bulk twisting are coupled as geometry optimizations with rigid in silico benzil molecules, described above, constrained the twisting observed. Below we discuss these two options.

Surface stress and surface reconstruction. The energy of broken chemical bonds at crystal surfaces and interfaces leads to surface energy and surface stress. Surface stresses are known to change unit cell dimensions in nanocrystals ${ }^{54}$ and represent a driving force for the reconstruction of metal and semiconductor crystal surfaces. ${ }^{48,55.56}$ On the other hand, bona fide examples of surface reconstruction in molecular crystals are few and far between. Researchers have considered differences in dimensions of molecular crystal surface cells established by AFM and those computed from crystal structures. ${ }^{57,58}$ They have recently focused on organic semiconductor crystals in thin films, because such "2D" crystals have large surfaces. 59,60 However, generally, surface reconstruction (or relaxation or surface-induced polymorphism, among other names) is small and difficult to detect. Roberts, with coworkers, surmised that the differences between the gas phase, and ground state conformation of benzophenone and its crystal structure conformation could account for the unexpected morphologies of solution-grown crystals via reconfiguration of surface molecules. ${ }^{61}$ Later, he and others showed by simulation, with support from XANES spectroscopy, that the molecules on the $\{0001\}$ surfaces of benzil were distorted from their crystal conformations. ${ }^{62,63}$ In fact, these computations suggested to us the possibility of testing surface stresses as a cause of crystal twisting, and directed us to our emphasis on benzil here, among the innumerable other sometimes twisted crystals we might have selected. However, we did not observe comparable changes in conformation at benzil \{0001\} surfaces with the GAFF or CVFF force fields, under geometry optimization or molecular dynamics simulation. No significant evidence for surface reconstruction was observed when simulating the infinite surface of benzil crystal under periodic boundary conditions. Albeit, our computational models are quite different from those used almost 25 years ago. We did, however, see conformational changes on the $\{10 \overline{1} 0\}$ surfaces in nanocrystals, as described above, with concomitant twisting. 
Although surface reconstruction can reduce surface stress, the remaining stress is significant ${ }^{48}$ and the presence of reconstruction indicates a high level of surface stresses. A lot of publications consider effect of crystal size on unit cell dimensions and evaluate surface stress in metals, semiconductors, oxides, and salts. ${ }^{54}$ Much fewer studies analyze molecular crystals and almost nothing is done about effect of surface stress on crystal morphology. Stresses arising from surfaces have long been implicated in the twisting of lamellae of polymers. ${ }^{64,65,66,67,68}$ Anisotropic surface stresses of -0.27 and $-0.4 \mathrm{~N} / \mathrm{m}$ calculated for the folding surfaces of polyethylene ${ }^{69}$ were in agreement with the values of -0.1 to $-0.2 \mathrm{~N} / \mathrm{m}$ experimentally determined for $n$-paraffins and polyethylene. ${ }^{70}$ These values allowed authors to correctly estimate the twist intensity in poly $\left(R\right.$-3-hydroxybutyrate) copolymers. $^{71}$

Using approach used in our previous publication, ${ }^{25}$ we developed a model to predict effect of crystal size on the surface stress induced twisting (see Supporting Information text). Figure S6 shows that for the crystals comparable in size with benzil crystals used in our simulations this effect can be roughly approximated by a power function $P \propto w^{1.3}$, which is not consistent with the computational results (eq. (1)). This shows that surface stress is unlikely to be the sole reason for benzil twisting revealed in our simulations.

Intrinsic deformations and geometric frustration. Given a bulk crystal with translational symmetry along all three dimensions, we can investigate how it behaves if it was cut into pieces. The crystal obviously begins in a state of mechanical equilibrium and thus in local stress balance. We expect this stress to vanish as we make the elements smaller and the intrinsic stress will become increasing manifest. The construction of a nanocrystal is in effect a computational liberation of the residual stresses in a straight, bulk crystal. An examination of this process was explored analytically and by experimental mathematics for homogeneous media. ${ }^{28}$ The extent to which these ideas can be extended to molecular suprastructures which are inhomogeneous (atomistic, and built of molecules of varying conformations on the interior and exterior) remains to be established. Nevertheless, computations herein are not inconsistent with a homogeneous body with an intrinsic local twist that has untwisted because it has conformed to a point lattice in order to grow.

In chemistry, there are many nanoscale supramolecular structures that are helicoidal and sizelimited. Because untwisting is a prerequisite for further growth - otherwise structures would 
become increasingly baroque with their growing cross sections - such materials are often sizelimited. Nanoscale structures made from chiral components, such as amyloid fibrils, are twisted, ${ }^{72}$ and for a long time, only twisted amyloid fibrils were known. However, it was recently shown that when fibers reach a critical width, they straighten and become translationally periodic. ${ }^{73,74,75}$ Some crystals may also be able to take advantage of the thermodynamic driving force of crystal growth once they straighten up. Benzil may be one of such crystals. Indirectly this connection is supported by the $P(w)$ scaling law. It was shown theoretically ${ }^{28}$ that as the number of coherently aligned twisted fibers increases, the twist intensity of the ensemble decreases with the same scaling law, $P \propto w^{2}$, as observed in our simulations (eq. (1)).

Correspondence with mesoscale twisting. Can twisting of mesoscopic (100 nm to $2 \mu \mathrm{m}$ in cross section) crystals - and even macroscopic crystals - observed in innumerable instances, be connected to intrinsic twisting of their nanocrystals? Might there be some mechanism whereby the intrinsic twist at the nanoscale is propagated, and preserved in part, as for much larger structures, thereby accounting for the phenomenologically very large number of highly crystalline compounds that grow as helicoidal fibers?

According to eq. (1), the twist period observed in benzil banded spherulites (Figure $1 \mathrm{~d}$ ) $P \approx 0.6$ $\mathrm{mm}$ corresponds to the crystal thickness $2 w \approx 0.44 \mu \mathrm{m}$, which falls in the range of typical thickness of fibers in banded molecular spherulites $(0.02-1 \mu \mathrm{m})$. On the other hand, the twist decay is proportional to the cross-sectional area, while for the vapor-grown hippuric acid crystals as well as some other materials pitch is roughly proportional to width, $P \propto w .{ }^{25,26} \mathrm{~A}$ possible reason for this deviation is plastic relaxation of elastic stress ${ }^{17,25,26}$ not considered in our geometry optimization procedure. As a crystal is deformed elastically as it grows, some part of the deformation becomes permanent (i.e. plastic) if the stress is high enough. Plastic stress relaxation may slow down decrease of the twist intensity, changing the scaling exponent from 2 to 1 .

Why are molecular crystals ever straight? Aggregates of molecules can associate with one another without restrictions on rotational symmetry. But as the aggregates get larger, as a crystal nucleus begins to grow, there are severe symmetry restrictions that exert themselves. Only rotational symmetries of order 1, 2, 3, 4, or 6 are permitted (quasicrystals notwithstanding). In other words, molecules have to accommodate themselves into predetermined symmetries that are 
consistent with point lattices having long range translational symmetry. If molecules become part of a crystal, they must make a compromise between the best interactions with their nearest neighbors, and what they must do in order to form a structure that is conformable to a lattice. From such a perspective, subvolumes within some crystals might be viewed as bodies under elastic stress. Moreover, for such a system, there is no stress free configuration.

Rybchintski and coworkers have pioneered the use of cryogenic transmission electron microscopy to observe molecular crystal nuclei (nanocrystals) growing in situ. ${ }^{76,77,78}$ There is every expectation that this body of work will expand rapidly in the near term. In aggregate, these studies show that many very small molecular crystals adopt their mature polyhedral forms in stages. In the interim, disordered condensates, as well as bent and twisted fibrils, assemble stepwise to form that which we recognize as a crystal. In other words, developing evidence indicates that very small crystalline (highly organized) materials are not polyhedral. Translational symmetry is not present in the initial stages of some crystallizations. It is something that crystals composed of weakly-bonded molecules sometimes grow into. After formation, such primary nanocrystal may still have enough flexibility to grow as non-classical twisted object and become straight and fully "classical" only as its size becomes sufficiently large.

Whether the best question is "Why are crystals twisted?" or "Why are crystals straight?" is an argument about forces that simultaneously have the characteristics of both a chicken and an egg. Some of the ubiquitous twisted crystals that we have observed may be "chickens" (something happened during their development), some others may be "eggs" (they were born that way).

\section{CONCLUSION}

We have found through simulation that some molecular crystals can twist spontaneously at the nanoscale. Twisting of benzil nanocrystals was studied in greatest detail. We proposed that this intrinsically twisted morphology may result from the surface stress due to the difference in the bonding patterns and molecular conformations on the surface and in the bulk. Alternatively, intrinsic stresses in crystal structures with long-range translational symmetry may be liberated at small sizes, thereby causing deformations at free surfaces where molecules are not constrained. Bulk and surface deformations are surely coupled, but which drives the other? As crystals gets larger in silico, they gradually untwist, hewing towards a 3D periodic crystal. Comparison of the 
twist intensity versus crystal size favors the dominance of the intrinsic stress mechanism. On a more general level, this is suggestive of a process whereby translational symmetry is a compromised accepted by mature crystals. There nevertheless remains a chasm between twisting of nanocrystals in molecular simulations and crystal twisting observed in optical banded spherulites on the mesoscale.

\section{Methods}

Optimizations and MD simulations were performed with LAMMPS (Large-scale Atomic/Molecular Massively Parallel Simulator) code. ${ }^{79}$ Both the Generalized Amber Forcefield (GAFF) ${ }^{44,45}$ and Consistent Valence Forcefield $(\mathrm{CVFF})^{80}$ were applied to simulate benzil crystals. For GAFF, the partial atomic charges were derived from a RESP calculation at the HF/6-31G* level of theory using the online R.E.D. web service. ${ }^{81,82,83,84}$ The partial atomic charges were from the default CVFF force field. The nanocrystals were placed in the center of an orthogonal simulation box. Periodic boundary conditions were removed as in our previous work. ${ }^{85}$ Electrostatic interactions were treated using full-pairwise summation by setting the interaction truncation cut-off to be larger than the length of the nanocrystal, such that the interactions between every pair of atoms in the system was calculated. The twisting is not an activated process and the rods assembled from crystallographic coordinates twisted spontaneously. All structures were initially optimized using a conjugate gradient minimization with relative energy tolerance of $10^{-8}$, as implemented in LAMMPS. The optimized structure was further relaxed using room temperature (300K) MD simulation with $1 \mathrm{fs}$ timestep. The temperature was controlled using a Langevin thermostat. The pitch was monitored during the simulation and the simulation was stopped when constant over time for at least 0.02 $\mathrm{ns}$, and the trajectory was recorded every $1 \mathrm{ps}$. The pitch in Figure $3 \mathrm{a}$ is the value averaged over the last 50 frames in the trajectory. For the torsion angle analysis reported in Figure 4, the structures were equilibrated for an extra $1 \mathrm{~ns}$ and the final results were averaged over the last 300 frames. Predefined twists shown in Figures 3 and 5 were imposed using the General Utility Lattice Program (GULP) code and the structures were relaxed by conjugate gradient minimization. Phonon frequency analysis was also calculated using GULP. ${ }^{36,37}$

In the process of choosing force fields for benzil, we calculated the phonon spectra of the trigonal, room temperature phase ${ }^{86}$ and the monoclinic low temperature phase ${ }^{87}$ Here, phonon properties for benzil unit cells were analyzed by calculating the eigenvalues of the dynamical matrix, the mass-weighted second derivative of the energy as a function of atomic displacement. A structure is an energy minimum if all the phonon frequencies are real whereas phonons with imaginary frequencies indicate dynamical instabilities. Two imaginary frequencies were computed during the optimization of the periodic trigonal crystal structure with both GAFF and CVFF force fields. On the other hand, the monoclinic structure had no 
imaginary frequencies. This finding is consistent with the monoclinic crystal structure being the stable form at low temperatures, as observed experimentally, while the trigonal structure represents the dynamic average over multiple equivalent minima, as observed above the phase transition temperature. The latter is best described as a hypertransition state with soft vibrations characteristic of a system subject to phase transformation.

The flexibility of molecules was controlled by adjusting the torsional parameters in the force field. The torsional potential in GAFF is calculated using the following equation

$$
E=K[1+\cos (n \vartheta-d)]
$$

where $K$ is the torsional force constant, $\vartheta$ is the torsion angle, $d$ is the phase angle, $n$ is the multiplicity. The flexibility of the benzil molecule is mainly controlled by the torsion angle of CA-C-C-CA, which contributes to the rotations of the phenyl rings with respect to carbonyl groups, and $\mathrm{O}=\mathrm{C}-\mathrm{C}=\mathrm{O}$ dihedrals, which represent the rotation of two $\mathrm{C}=\mathrm{O}$ bonds with respect to each other. Hence, in our work, the molecular flexibility is controlled by the $K$ value. The $d$ parameter was also changed to ensure that the minimum energy conformation was that of the crystallographic benzil molecule $\left(\Psi=107^{\circ}\right)$. The gas phase equilibrium torsional angle $\left(116.3^{\circ}\right)$ was determined by optimization at the $\omega \mathrm{B} 97 \mathrm{X}-\mathrm{D} 3 / \mathrm{def} 2-$ QZVPP level of theory ${ }^{88}$ using the ORCA code. ${ }^{89}$

\section{- ASSOCIATED CONTENT \\ S Supporting Information}

The Supporting Information is available free of charge on the ACS Publications website at DOI: XXX. Crystallographic Information File (CIF) for $P 3_{1} 21$ benzil; Partial atomic charges and mass; Force field parameters; Lattice parameters comparisons between experimental crystal structure and calculated structure using GAFF at room temperature/100 K; Right-handed twisted nanocrystals for $P 3_{2} 21$ benzil; Twisting angle characterization; Twisting angle comparison among benzil nanocrystals with different lengths; Molecular conformation analysis for 4phenoxyaniline and 4,4'-dimethylbenzophenone.

\section{- AUTHOR INFORMATION}

Corresponding Authors

*E-mail: J.Gale@ curtin.edu.au

*E-mail: andrew.rohl@curtin.edu.au

*E-mail: bart.kahr@nyu.edu 
ORCID

Alexander G. Shtukenberg: 0000-0002-5590-4758

Leslie Vogt-Maranto: 0000-0002-7006-4582

Bart Kahr: 0000-0002-7005-4464

Paolo Raiteri: 0000-0003-0692-0505

Andrew Rohl: 0000-0003-0038-2785

Julian Gale: 0000-0001-9587-9457

Notes

The authors declare no competing financial interests.

\section{ACKNOWLEDGMENTS}

This work was primarily supported by the New York University Materials Research Science and Engineering Center (MRSEC) program of the National Science Foundation under award number DMR-1420073. Funding was also provided by the National Science Foundation and the U.S.Israel Binational Science Foundation (grants DMR-1608374, 2003968 and NSF/DMR-BSF 2015670, respectively). This work was supported by computational resources provided by High Performance Computing resources at New York University, as well as from the Pawsey Supercomputing Centre and National Computational Infrastructure. The Australian Research Council is thanked for support via fellowships to PR (FT130100463) and JG (FL180100087).

\section{- REFERENCES}

(1) International Union of Crystallography, Report of the executive committee of 1991. Acta Crystallogr. 1992, A48, 922-946.

(2) Saha, S.; Desiraju, G. R. Crystal engineering of hand-twisted helical crystals. J. Am. Chem. Soc. 2017, 13, 1975-1983.

(3) Saha, S.; Desiraju, G. R. A hand twisted helical crystal based solely on hydrogen bonding, Chem. Commun. 2017, 53, 6371-6374.

(4) Zhu, L.; Al-Kaysi, R. O.; Bardeen, C. J. Reversible photoinduced twisting of molecular crystal microribbons. J. Am. Chem. Soc. 2011, 133, 12569-12575.

(5) Kim, T.; Zhu, L.; Mueller, L. J.; Bardeen, C. J. Dependence of the solid-state photomechanical response of 4-chlorocinnamic acid on crystal shape and size. CrystEngComm 2012, 14, 7792-7799.

(6) Kim, T.; Zhu, L.; Mueller, L. J.; Bardeen, C. J. Mechanism of photoinduced bending and twisting in crystalline microneedles and microribbons composed of 9-methylanthracene. J. Am. Chem. Soc. 2014, 136, 6617-6625. 
(7) Kitagawa, D.; Nishi, H.; Kobatake, S. Photoinduced twisting of a photochromic diarylethene crystal. Angew. Chem. Int. Ed. 2013, 52, 9320-9322.

(8) Kitagawa, D. Tsujioka, H.; Tong, F.; Dong, X.; Bardeen, C. J.; Kobatake, S. Control of photomechanical crystal twisting by illumination direction. J. Am. Chem. Soc. 2018, 140, 4208-4212.

(9) Rai, R.; Krishnan, B. P.; Sureshan, K. M. Chirality-controlled spontaneous twisting of crystals due to thermal topochemical reaction. Proc. Natl. Acad. Sci. U. S. A. 2018, 115, 2896-2901.

(10) Gupta, P.; Karothu, D. P.; Ahmed, E.; Naumov, P.; Nath, N. K. Thermally twisted, photobendable, elastically deformable, and self-healable soft crystals. Angew. Chem. Int. Ed. 2018, 57, 8498-8502.

(11) Rai, R.; Krishnan, B. P.; Sureshan, K. M. Chirality-controlled spontaneous twisting of crystals due to thermal topochemical reaction. Proc. Natl. Acad. Sci. U. S. A. 2018, 115, 2896-2901.

(12) Naumov, P.; Chizhik, S.; Panda, M. K.; Nath, N. K.; Boldyreva, E. Mechanically responsive molecular crystals. Chem. Rev. 2015, 115, 12440-12490.

(13) Wallerant, F. C. R. Hebd. Séances Acad. Sci. 1906, 143, 555-557.

(14) Wallerant, F. C. R. Hebd. Séances Acad. Sci. 1906, 143, 1169-1170.

(15) Wallerant, F. Bull. Soc. Fr. Mineral. 1907, 30, 43-60.

(16) Bernauer, F. “Gedrillte” Kristalle; Gebrüder Borntraeger: Berline, 1929.

(17) Shtukenberg, A. G.; Punin, Y. O.; Gujral, A.; Kahr, B. Growth actuated bending and twisting of single crystals. Angew. Chem. Int. Ed. 2014, 53, 672-699.

(18) Suda, J.; Matsushita, M. Chirality change in helical crystals of potassium dichromate. $J$. Phys. Soc. Jpn. 2004, 73, 300-302.

(19) Zorz, M. Quarz-Gwindel. Mineralien der Welt. 2009, 20, 30-46.

(20) Cui, X.; Rohl, A. L.; Shtukenberg, A. G.; Kahr, B. Twisted aspirin crystals. J. Am. Chem. Soc. 2013, 135, 3395-3398.

(21) Zhu, Q.; Shtukenberg, A. G.; Carter, D. J.; Yu, T.-Q.; Yang, J.; Chen, M.; Raiteri, P.; Oganov, A. R.; Pokroy, B.; Polishchuk, I. et al. Resorcinol crystallization from the melt: a new ambient phase and new "riddles". J. Am. Chem. Soc. 2016, 138, 4881-4889.

(22) Yang, J.; Hu, C. T.; Shtukenberg, A. G.; Yin, Q.; Kahr, B. L-Malic acid crystallization: polymorphism, semi-spherulites, and polarity. CrystEngComm 2018, 20, 1383-1389.

(23) Shtukenberg, A. G.; Punin, Y. O.; Gunn, E.; Kahr, B. Spherulites. Chem. Rev. 2012, 112, 1805-1838.

(24) Shtukenberg, A. G.; Freundenthal, J.; Gunn, E.; Yu, L.; Kahr, B. Glass-crystal growth mode for testosterone propionate. Cryst. Growth Des. 2011, 11, 4458-4462.

(25) Shtukenberg, A. G.; Gujral, A.; Rosseeva, E.; Cui, X.; Kahr, B. Mechanics of twisted hippuric acid crystals untwisting as they grow. CrystEngComm 2015, 17, 8817-8824. 
(26) Shtukenberg, A. G.; Freudenthal, J.; Kahr, B. Reversible twisting during helical hippuric acid crystal growth. J. Am. Chem. Soc. 2010, 132, 9341-9349.

(27) Shtukenberg, A. G.; Cui, X.; Freudenthal, J.; Gunn, E.; Camp, E.; Kahr, B. Twisted mannitol crystals establish homologous growth mechanisms for high polymer and small molecule ring banded spherulites. J. Am. Chem. Soc. 2012, 134, 6354-6364.

(28) Haddad, A.; Aharoni, H.; Sharon, E.; Shtukenberg, A. G.; Kahr, B.; Efrati, E. Twist renormalization in molecular crystals driven by geometric frustration. Soft Matter 2019, 15, 116-126.

(29) Becker, K.; Rose, H. X-ray spectroscopy on organic compounds. Z. Phys. 1923, 14, 369373.

(30) Allen, N. C. B. The crystal structure of benzil. Phil. Mag. 1927, 3, 1037-1040.

(31) Brown, C. J.; Sadanaga, R. The crystal structure of benzil. Acta Cryst. 1965, 18, 158-164.

(32) Gabe, E. J.; Page, T. L.; Lee, F. L. The structure of 2,2',4,4',6,6'-Hexa-tert-butylbenzil. Acta Cryst. 1981, B37, 197-200.

(33) More, M.; Odou, G.; Lefebvre, J. Structure determination of benzil in its two phases. Acta Cryst. 1987, B43, 398-405.

(34) Yadav, H.; Sinha, N.; Kumar, B. Growth and characterization of piezoelectric benzil single crystals and its application in microstrip patch antenna. CrystEngComm 2014, 16, 10700-10710.

(35) Sankaranarayanan, K.; Ramasamy, P. Microtube-Czochralski technique (uT-CZ): A novel way of seeding the melt to grow bulk single crystal. J Cryst. Growth 1998, 193, 252-256.

(36) Gale, J. D.; Rohl, A. L. The general utility lattice program (GULP). Mol Simul. 2003, 29, 291-341.

(37) Gale, J. D. GULP: Capabilities and prospects. Z. Kristallogr. 2005, 220, 552-554.

(38) Calswell, C. C.; Le Févre, R. J. W. Evidence for a skew structure of benzil. J. Chem. Soc. 1939, 1614-1622.

(39) Knaggs, I. E.; Lonsdale, K. Structure of benzil. Nature 1939, 143, 1023-1024.

(40) Morantz, D. J.; Wright, A. J. C. Structure of the excited states of benzil and related dicarbonyl molecules. J. Chem. Phys. 1971, 54, 692-696.

(41) Shen, Q.; Hagen, K. Gas-phase molecular structure and conformation of benzil as determined by electron diffraction, J. Phys. Chem. 1987, 91, 1357-1360.

(42) Pawelka, Z.; Koll, A.; Zeegers-Huyskens, T. Solvent effect on the conformation of benzil, J. Mol. Struct. 2001, 597, 57-66.

(43) Bhattacharya, B.; Jana, B.; Bose, D.; Chattopadhyay, N. Multiple emissions of benzil at room temperature and $77 \mathrm{~K}$ and their assignments from ab initio quantum chemical calculations. J. Chem. Phys. 2011, 134, 044535.

(44) Wang, J.; Wolf, R. M.; Caldwell, J. W.; Kollman, P. A.; Case, D. A. Development and testing of a general amber force field. J. Comput. Chem. 2004, 25, 1157-1174. 
(45) Nemkevich, A.; Bürgi, H.B.; Spackman, M. A.; Corry, B. Molecular dynamics simulations of structure and dynamics of organic molecule crystals. Phys. Chem. Chem. Phys. 2010, 12, 14916-14929.

(46) Shabbir, M.; Akhter, Z.; Ahmad, I.; Ahmed, S.; Bolte, M.; McKee, V. Synthesis and bioelectrochemical behavior of aromatic amines. Bioorg. Chem. 2017, 75, 224-234.

(47) Soqaka, A. A.; Esterhuysen, C.; Lemmerer, A. Prevalent polymorphism in benzophenones. Acta Crystallogr. 2018, C74, 465-471.

(48) Ibach, H. The role of surface stress in reconstruction, epitaxial growth and stabilization of mesoscopic structures. Surf. Sci. Rep. 1997, 29, 193-263.

(49) Cammarata, R. C.; Sieradzki, K. Surface and interface stresses. Annu. Rev. Mater. Sci. 1994, 24, 215-234.

(50) Niv, I.; Efrati, E. Geometric frustration and compatibility conditions for two-dimensional director fields, Soft Matter 2018, 14, 424-431.

(51) Hall, D. M. How geometric frustration shapes twisted fibres, inside and out: competing morphologies of chiral filament assembly. Interface Focus 2017, 7, 20160140.

(52) Grason, G. M. Geometrically frustrated assemblies. J. Chem. Phys. 2016, 145, 110901.

(53) Grason, G. M. Chiral and achiral mechanisms of self-limiting assembly. Soft Matter 2020, 16, 1102-1116.

(54) Diehm, P. M.; Ágoston, P.; Albe, K. Size-dependent lattice expansion in nanoparticles: Reality or anomaly? ChemPhysChem 2012, 13, 2443-2454.

(55) Duke, C. B. Semiconductor surface reconstruction: The structural chemistry of twodimensional surface compounds. Chem. Rev. 1996, 96, 1237-1259.

(56) Giessibl, F. J. Atomic resolution of the silicon (111)-(7×7) surface. Science 1995, 267, 68-71.

(57) Ward, M. D. Bulk crystals to surfaces: Combining X-ray diffraction and atomic force microscopy to probe the structure and formation at crystal interfaces. Chem. Rev. 2001, $101,1697-1725$.

(58) Overney, R. M.; Howald, L.; Frommer, J.; Meyer, E.; Brodbeck, D.; Güntherodt, H.-J. Molecular surface structure of organic crystals observed by atomic force microscopy. Ultramicroscopy 1992, 42-44, 983-988.

(59) Morisaki, H.; Koretsune, T.; Hotta, C.; Takeya, J.; Kimura, T.; Wakabayashi, Y. Large surface reconstruction in the organic semiconductor tetracene. Nat. Commun. 2014, 5, 5400 .

(60) Wakabayashi, Y.; Nakamura, M.; Sasaki, K.; Maeda, T.; Kishi, Y.; Ishii, H.; Kobayashi, N.; Yanagisawa, S.; Shimo, Y.; Kubozono, Y. Surface structure of organic semiconductor [n]phenacene single crystals. J. Am. Chem. Soc. 2018, 140, 14046-14049. 
(61) Roberts, K.; Docherty, R.; Bennema, P.; Jetten, L. A. M. J. The importance of considering growth-induced conformational change in predicting the morphology of benzophenone. J. Phys. D: Appl. Phys. 1993, 26, B7-B21.

(62) Hastie, G. P.; Johnstone, J.; Roberts, K. J.; Walker, E. M. The effect of surface molecular conformational change on morphology: A study of benzil (0001). Mol. Cryst. Liq. Cryst. Sci. Tech. Sect. A. 1996, 277, 29-37.

(63) Hastie, G. P.; Johnstone, J.; Walker, E. M.; Roberts, K. J. Direct evidence for surface reconstruction on organic solid surfaces: Benzil (0001). J. Chem. Soc. Perkin Trans. 1996, 2, 2049-2050.

(64) Lotz, B.; Cheng, S. Z. D. A critical assessment of unbalanced surface stresses as the mechanical origin of twisting and scrolling of polymer crystals. Polymer 2005, 46, 577610.

(65) Bassett, D. C. A critical assessment of unbalanced surface stresses: Some complementary considerations. Polymer 2006, 47, 3263-3266.

(66) Keith, H. D.; Padden, Jr., F. J. Twisting orientation and the role of transient states in polymer crystallization. Polymer 1984, 25, 28-42.

(67) Crist, B.; Schultz, J. M. Polymer spherulites: A critical review. Prog. Polym. Sci. 2016, $56,1-63$.

(68) Lovinger, A. Twisted crystals and the origin of banding in spherulites of semicrystalline polymers. Macromolecules 2020, 53, 741-745.

(69) Hütter, M.; in 't Veld, P. J.; Rutledge, G. C. Polyethylene $\{201\}$ crystal surface: Interface stresses and thermodynamics. Polymer 2006, 47, 5494-5504.

(70) Cammarata, R. C.; Eby, R. K. Effects and measurement of internal surface stresses in materials with ultrafine microstructures. J. Mater. Res. 1991, 6, 888-890.

(71) Ye, H.; Wang, J.; Tang, S.; Xu, J.; Feng, X.; Guo, B.; Xie, X.; Zhou, J.; Li, L.;Wu, Q. et al. Surface stress effects on the bending direction and twisting chirality of lamellar crystals of chiral polymer. Macromolecules 2010, 43, 5762-5770.

(72) Volpatti, L. R.; Vendruscolo, M.; Dobson, C. M.; Knowles, T. P. J. A clear view of polymorphism, twist, and chirality in amyloid fibril formation. ACS Nano 2013, 7, 10443-10448.

(73) Knowles, T. P. J.; De Simone, A.; Fitzpatrick, A. W.; Baldwin, A.; Meehan, S.; Rajah, L.; Vendruscolo, M.; Welland, M. E.; Dobson, C. M.; Terentjev, E. M. Twisting transition between crystalline and fibrillar phases of aggregates peptides. Phys. Rev. Lett. 2012, 109, 158101.

(74) Reynolds, N. P.; Adamcik, J.; Berryman, J. T.; Handschin, S.; Zanjani, A. A. H.; Li, W.; Liu, K.; Zhang, A.; Mezzenga, R. Competition between crystal and fibril formation in molecular mutations of amyloidogenic peptides. Nat. Commun. 2017, 8, 1338.

(75) Adamcik, J.; Mezzenga, R. Amyloid polymorphism in the protein folding and aggregation energy landscape. Angew. Chem. Int. Ed. 2018, 57, 2-15. 
(76) Shahar, C.; Dutta, S.; Weissman, H.; Shimon, L. J. W.; Ott, H.; Rybtchinski, B. Precrystalline aggregates enable control over organic crystallization in solution. Angew. Chem. Int. Ed. 2016, 55, $179-182$.

(77) Tsarfati, Y.; Rosenne, S.; Weissman, H.; Shimon, L. J. W.; Gur, D.; Palmer, B. A.; Rybtchinski, B. Crystallization of organic molecules: Nonclassical mechanism revealed by direct imaging. ACS Cent. Sci. 2018, 4, 1031-1036.

(78) Bronshtein, I.; Weissman, H.; Kaplan-Ashiri, I.; Rybtchinski, B. Crystallization of small organic molecules in a polymer matrix: Multistep mechanism enables structural control. Small 2019, 15, 1902936.

(79) Plimpton, S. Fast parallel algorithms for short-range molecular dynamics. J. Comput. Phys. 1995, 117, 1-19.

(80) Dauber-Osguthorpe, P.; Roberts, V. A.; Osguthorpe, D. J.; Woff, J.; Genest, M.; Hagler, A. T. Structure and energetics of ligand binding to proteins: Escherichia coli dihydrofolate reductase-trimethoprim, a drug-receptor system. Proteins: Struct. Funct. Genet. 1988, 4, 31-47.

(81) Vanquelef, E., Simon, S.; Marquant, G.; Garcia, E.; Klimerak, G.; Delepine, J. C.; Cieplak, P.; Dupradeau, F.-Y. R.E.D. Server: a web service for deriving RESP and ESP charges and building force field libraries for new molecules and molecular fragments. Nucl. Acids Res. 2011, 39, W511-W517.

(82) Wang, F.; Becker, J. P.; Cieplak, P.; Dupradeau, F. Y. R.E.D. Python: Object oriented programming for Amber force fields. Université de Picardie - Jules Verne, Sanford Burnham Prebys Medical Discovery Institute, Nov. 2013.

(83) Dupradeau, F. Y.; Pigache, A.; Zaffran, T.; Savineau, C.; Lelong, R.; Grivel, N.; Lelong D.; Rosanski, W.; Cieplak, P. The R.E.D. tools: Advances in RESP and ESP charge derivation and force field library building. Phys. Chem. Chem. Phys. 2010, 12, 78217839.

(84) Bayly, C.I.; Cieplak, P., Cornell, W.; Kollman, P. A. A well-behaved electrostatic potential based method using charge restraints for deriving atomic charges: The RESP model. J. Phys. Chem. 1993, 97, 10269-10280.

(85) Li, C.; Shtukenberg, A. G.; Carter, D. J.; Cui, X.; Olson, I.; Rohl, A. L.; Gale, J. D.; Raiteri, P.; Kahr, B. Timoshenko bending and Eshelby twisting predicted in molecular nanocrystals, J. Phys. Chem. C, 2018, 122, 25085-25091.

(86) Brown, C. J.; Sadanaga, R. The crystal structure of benzil. Acta Crystallogr. 1965, 18, $158-164$.

(87) More, M.; Odou, G.; Lefebvre, J. Structure determination of benzil in its two phases. Acta Crystallogr. 1987, B43, 398-405.

(88) Lin, Y.-S.; Li, G.-D.; Mao, S.-P.; Chai, J.-D. Long-range corrected hybrid density functionals with improved dispersion corrections. J. Chem. Theory Comput. 2013, 9, 263-272.

(89) Neese, F. The ORCA Program System. WIREs Comput. Mol. Sci. 2012, 2, 73-78. 